\title{
Medicine in the digital age
}

\author{
As Nature Medicine celebrates its 25th anniversary, we bring you a special Focus on Digital Medicine that highlights \\ the new technologies transforming medicine and healthcare, as well as the related regulatory challenges ahead.
}

$\int$ igital medicine, defined as the use of digital tools to upgrade the practice of medicine to one that is high definition and individualized, holds promise in revolutionizing healthcare and well-being. At the core of this revolution is the development of technological solutions to monitor, process and integrate vast amounts of data at the individual and population levels to help address the health problems and challenges faced by patients, clinicians and health systems alike. This new digital age of medicine has the potential to democratize access to care and empower patients to engage with their health in a truly preventive way. It also holds the promise of helping clinicians to navigate the increasing volume and complexity of patients' data in a cost-efficient and timesaving manner. This issue's Focus on Digital Medicine represents a collective effort of our editorial team to bring together new research, commissioned articles and features that explore opportunities and challenges in this growing field.

Artificial intelligence (AI), and in particular deep learning, is among the leading technological tools beginning to be used in the interpretation of medical images and electronic health records. These advances are discussed in a Perspective by Jeff Dean and colleagues ('A guide to deep learning in healthcare'). AI tools are speeding up diagnoses, guiding therapeutic choices and optimizing patient experience. Last April, in a milestone approval (https:// www.fda.gov/newsevents/newsroom/ pressannouncements/ucm604357.htm), the US Food and Drug Administration (FDA) granted approval to an artificial intelligence-based device to detect certain diabetes-related eye problems. It was the agency's first market approval of a medical device that performs a screening test and provides a referral to a specialist without the need for a clinician to interpret the image or results. The agency has also announced its intention to use cost-effective strategies and big data to accelerate the regulatory path of clinical trials, medical product development and innovations in AI. The Pre-Certification Pilot Program (https:// www.fda.gov/medicaldevices/digitalhealth/ digitalhealthprecertprogram/default.htm), one of the outcomes of this FDA initiative, is what enabled Apple to quickly receive clearance for use of its electrocardiography app (a feature of the new Apple watch), which provides over-the-counter information for identification of certain cardiac arrhythmias in users of the hightech watch. Another nine tech companies (https://www.fda.gov/newsevents/ newsroom/pressannouncements/ ucm577480.htm) were also preselected to be part of the pilot phase of this program. It's fair to assume that we will be seeing new devices approved in the very near future. Check out the Perspective by Kang Zhang and colleagues for an update on the regulatory environment of AI-based technologies across the globe ('The practical implementation of artificial intelligence technologies in medicine').

As noted in Eric Topol's Review ('Highperformance medicine: the convergence of human and artificial intelligence'), although the benefits of this digital revolution are easy to point out-such as improvements in accuracy, productivity, workflows and access - the magnitude of the challenges it creates is harder to predict. Over-the-counter use of medical devices to detect conditions that normally would require assessment by a physician could lead to overdiagnosis in the healthy population, increasing the burden handled by health care systems instead of relieving it. Moreover, there is still a paucity of prospective clinical studies assessing whether there is a net benefit of incorporating AI algorithms and devices in clinical practice. Other challenges in digital medicine, discussed by Glenn Cohen and colleagues ('Privacy in the age of medical big data'), include ensuring the privacy of health data and establishing proper consent and patient governance in data collection, as well as developing regulation to protect individuals against the use of personal data for discrimination. Finally, it is worth exploring how the human aspect of medicine-the bond between patient and physician-may be affected by the increasing use of technology in medical practice.

When Nature Medicine launched 25 years ago, the World Wide Web was only beginning to take off, and medicine had nothing to do with mobile phones and smartwatches. Now that we are in the throes of a digital revolution, it is worth putting all of its many dimensions under the microscope. The celebration of our 25th anniversary, however, is just at the beginning. Throughout the year we will be highlighting young to midcareer researchers who are changing the landscape of medicine as well as special features on how medicine has changed over the past 25 years ('Twentyfive ways clinical trials have changed in the last 25 years'). Last but not least, we want to thank our authors, reviewers and readers, who for the past two and a half decades have been our partners in making Nature Medicine a highly successful journal. We look forward to the next 25 years.

Published online: 7 January 2019 https://doi.org/10.1038/s41591-018-0322-1 